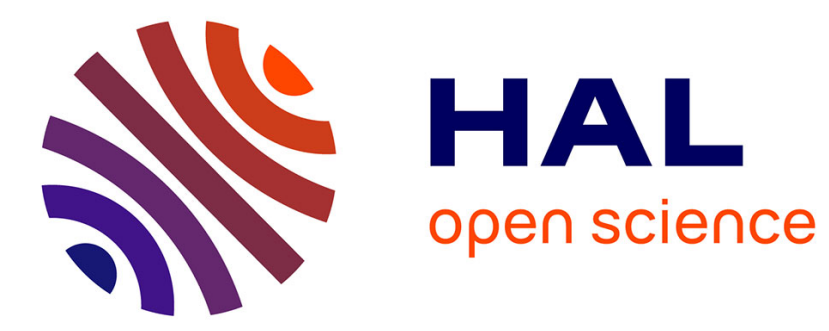

\title{
Peut-on augmenter l'efficacité de la thérapie cognitive et comportementale pour le trouble obsessionnel compulsif par un adjuvant informatique innovant ?

\author{
Margot Morgiève, K. N'diaye, A.-H. Clair, A. Pelissolo, L. Mallet
}

\section{- To cite this version:}

Margot Morgiève, K. N'diaye, A.-H. Clair, A. Pelissolo, L. Mallet. Peut-on augmenter l'efficacité de la thérapie cognitive et comportementale pour le trouble obsessionnel compulsif par un adjuvant informatique innovant ?. L'Encéphale, 2016, 10.1016/j.encep.2016.03.004 . hal-01311538

\section{HAL Id: hal-01311538 \\ https: / hal.sorbonne-universite.fr/hal-01311538}

Submitted on 4 May 2016

HAL is a multi-disciplinary open access archive for the deposit and dissemination of scientific research documents, whether they are published or not. The documents may come from teaching and research institutions in France or abroad, or from public or private research centers.
L'archive ouverte pluridisciplinaire HAL, est destinée au dépôt et à la diffusion de documents scientifiques de niveau recherche, publiés ou non, émanant des établissements d'enseignement et de recherche français ou étrangers, des laboratoires publics ou privés. 


\title{
Peut-on augmenter l'efficacité de la thérapie cognitive et comportementale pour le trouble obsessionnel compulsif par un adjuvant informatique innovant?
}

\author{
Margot Morgiève, $\mathrm{PhD}^{\mathrm{a}, \mathrm{b} *^{\bullet}}$; Karim N'Diaye, $\mathrm{PhD}^{\mathrm{a}, \mathrm{b}^{\bullet}}$; Anne-Hélène Clair, $\mathrm{PhD}^{\mathrm{a}}$; \\ Antoine Pelissolo, MD, PhD ${ }^{b, c}$; Luc Mallet, MD, PhD \\ a Équipe Behavior, Emotion, and Basal Ganglia, CNRS UMR 7225, Inserm UMRS 975, Université Pierre et Marie Curie \\ (UPMC), ICM (Institut du Cerveau et de la Moëlle épinière), CHU Pitié-Salpêtrière, Paris, France \\ ${ }^{b}$ Fondation FondaMental, Créteil, France \\ ' Pôle de psychiatrie, Hôpitaux Universitaires Henri-Mondor, AP-HP, UPEC, INSERM U955, Créteil, France \\ ${ }^{d}$ Centre d'Investigation Clinique, CHU Pitié-Salpêtrière, Paris, France \\ Adresse de correspondance : \\ Margot Morgiève \\ Hôpital de la Salpêtrière \\ ICM - Institut du Cerveau et de la Moelle épinière \\ 47-83, boulevard de l'Hôpital \\ 75651 PARIS Cedex 13 \\ 0157274395 \\ 0157274789 \\ margot.morgieve-mas@inserm.fr
}




\section{Résumé}

La thérapie cognitive et comportementale (TCC) est reconnue comme efficace pour soigner le trouble obsessionnel compulsif (TOC). Pour maximiser son efficacité, nous avons conçu, dans une démarche participative associant patients et thérapeutes, une TCC « expérimentale » définie par l'adjonction d'un outil psychopédagogique informatisé basé sur une tâche de vérification. Cette étude randomisée en double insu inclut 35 patients atteints de TOC de vérification suivis en TCC «standard » ou «expérimentale» sur le plan clinique et neurocomportemental grâce à une tâche originale de provocation de symptômes avec des photographies neutres, anxiogènes génériques et anxiogènes personnalisées. Les thérapeutes ont apprécié la dimension psychopédagogique de l'outil informatisé. Les deux TCC ont entraîné une amélioration des symptômes équivalente et significative et une diminution de l'anxiété induite par les photographies anxiogènes personnalisées. La réponse à mi-thérapie était prédictive de l'amélioration finale. L'outil informatisé peut fournir un adjuvant thérapeutique bien accepté même s'il n'augmente pas le gain thérapeutique. La tâche de provocation de symptômes utilisant des images personnalisées permit de mettre en évidence l'évolution parallèle des symptômes et des marqueurs neurocomportementaux au cours des TCC. Malgré la difficulté d'augmenter l'efficacité d'une thérapie fondée sur les preuves, les résultats observés à mi-thérapie suggèrent d'explorer précocement les ajustements possibles des stratégies thérapeutiques.

Cette étude sous promotion INSERM est enregistrée sous le titre "Modification of Cerebral Activity of Obsessive Compulsive Disorder (OCD) Patients During Cognitive and Behavioral Therapy (TOC TOC)", URL: http://clinicaltrials.gov/ct2/show/NCT01331876.

\section{Mots clefs}

Trouble obsessionnel compulsif ; Thérapie cognitive et comportementale ; Innovation thérapeutique ; Outil informatisé ; essais cliniques comme sujet 


\section{Can the efficacy of Behavioral and Cognitive Therapy for Obsessive Compulsive Disorder be augmented by innovative computerized adjuvant?}

\section{Summary}

Aim: Cognitive behavioral therapy (CBT) is recognized as an effective treatment for obsessive-compulsive disorder (OCD). To maximize its effectiveness, we designed an "experimental" CBT defined by the addition of a computerized psychoeducative tool.

Method: In a participative process involving patients through the French OCD association (AFTOC) meetings and therapists through methodological workshops, we built a therapeutic tool from an experimental checking task. This task, which had been published in earlier work, was adapted for its psychoeducative dimension.

We here report on a randomized double-blind trial included 35 patients with a moderate to severe OCD (Yale-Brown Obsessive-Compulsive Scale, YBOCS between 16 and 25) predominant checking symptoms, no comorbidities, and two-month stabilized or no treatment. Patients were randomly assigned to either "standard" versus "experimental" CBT. Both therapies were conducted by four CBT-experienced therapist specialized in OCD through weekly individualized sessions over three months. Therapy sessions of the experimental CBT were conducted as the standard CBT except for a short exercise with the computerized psychoeducative tool performed by the patient and debriefed with the therapist at the end of the sessions.

Patients were assessed before, during, after and six months after therapy using standard clinical tools and a neurobehavioral assessment based on an original symptom-provocation task with anxiety ratings including three types of photographs: neutral, generic inducing obsessions (e.g., doorknobs, electric wires,...) and personalized (taken by the patients in their own environment).

Results: Clinically, "standard" and "experimental" CBT resulted in a significant but equivalent improvement ( $48 \%$ vs $45 \%$ reduction of the Y-BOCS score; $P=0,36 ; d=0,12$ ).

Therapists were satisfied with the psychoeducative dimension of the computerized psychoeducative tool, but reported variable acceptance across patients. Patients appreciated its usability.

The clinical improvement was associated to a reduction of the task-induced anxiety $(r=0,42, p<0,05)$, especially towards personalized items $(-28,2 \%$ v. $-20,41 \%$ for generic and $-6,24 \%$ for neutral photographs, $P<0,001)$. Mid-therapy response level was predictive of the final improvement $(r=0,82, P<0,001)$.

Conclusion: The computerized tool may provide a well-accepted therapeutic adjuvant even though it doesn't improve the therapeutic effect. Using a personalized symptom-provocation task reveals the parallel evolution of symptoms and neurobehavioral markers through CBT.

Despite the difficulty of improving an evidence-based therapy, mid-therapy results call for investigating the possible adjustments of treatment strategies at an early stage.

Clinical trial: "Modification of Cerebral Activity of Obsessive Compulsive Disorder (OCD) Patients During Cognitive and Behavioural Therapy (TOC TOC)", URL: http://clinicaltrials.gov/ct2/show/NCT01331876.

\section{Keywords}

Obsessive Compulsive Disorder; Cognitive Behavioral Therapy; Therapeutic Research; Software Tools; Clinical trial as Topic 


\section{Introduction}

Le trouble obsessionnel-compulsif (TOC) est le quatrième trouble psychiatrique le plus fréquent, touchant 2-3\% de la population générale [1]. Le TOC est défini par la combinaison de A) les obsessions, qui sont des idées récurrentes et pénibles, et $B$ ) les compulsions, des comportements que le sujet se sent obligé d'effectuer pour prévenir ou réduire l'anxiété associée à des obsessions. Les traitements de première intention pour le TOC sont d'une part des médicaments (inhibiteurs de la recapture de la sérotonine), d'autre part la thérapie cognitive et comportementale (TCC) [2]. Cependant, 25 à $40 \%$ des patients présentent des symptômes persistants et des répercussions fonctionnelles durables [3], ce qui incite à chercher de nouveaux outils thérapeutiques.

Les progrès rapides de la technologie au cours des dernières décennies ont ouvert la voie à un large éventail d'innovations dans le domaine de la santé mentale [4] [5]. L'utilisation de l'ordinateur pour les patients atteints de TOC est particulièrement profitable [6]. Les technologies de la réalité virtuelle sont utilisées pour traiter les troubles anxieux [7]. L'introduction de tels médias a un impact positif sur le sentiment d'auto-efficacité et le sens de la maîtrise des patients, et peut améliorer l'observance du traitement [8]. Il semble donc pertinent de combiner ces dispositifs à des interventions cognitives et comportementales afin de maximiser leur efficacité.

Le développement d'alternatives thérapeutiques s'accompagne d'une volonté de mieux comprendre les dynamiques de l'évolution clinique et cérébrale des patients en cours de thérapie.

Selon les modèles neurocognitifs du TOC [9] [10], la partie cognitive de la TCC est conçue pour corriger les schémas cognitifs erronés à l'origine des obsessions. Cependant, les questions de savoir quand et comment les changements anatomo-fonctionnels induits par la TCC [11] se produisent n'ont pas été examinées de manière extensive [12]. Au niveau cérébral, ces processus impliqueraient notamment les cortex cingulaire antérieur et orbitofrontal [13] connus pour leur rôle dans le contrôle cognitif de l'émotion [14]. Leur mise en évidence repose sur des «tâches d'exposition » à des stimuli déclenchant les symptômes [15] dites aussi «tâches de provocation de symptômes " [16] pour les différencier de la procédure d'exposition (et de prévention de la réponse) utilisée en TCC [17]. Pour sensibiliser la détection des changements neurocognitifs, nous avons utilisé une tâche de provocation de symptômes que nous avons couplée avec des enregistrements en IRM fonctionnelle.

Le présent article ne traitera pas des résultats de l'IRM fonctionnelle (développés dans un article publié par ailleurs [18]). II s'intéressera (i) au processus de création d'un adjuvant thérapeutique dans le cadre de la TCC pour le TOC et (ii) aux résultats cliniques et comportementaux liés à cette intervention.

\section{Méthode}

\section{Les participants}

Soixante-six patients atteints de TOC (âgés de 18 à 65 ans) ont été sélectionnés via l'Association Française de patients atteints de TOC (AFTOC) et la CLinique d'Investigation de la Cognition et du Comportement (CLICC) à l'hôpital de la Pitié-Salpêtrière, pour participer à un essai clinique durant lequel ils devaient suivre trois mois de TCC. Une psychologue clinicienne (MM) a mené des entretiens individuels semi-structurés afin d'établir le diagnostic de TOC selon les critères du DSM-IV-TR et d'exclure des comorbidités (MINI 5.0.0) [19]. La sévérité des symptômes a été évaluée en utilisant la Yale-Brown Obsessive Compulsive Scale (Y-BOCS, [20]). L'anxiété et la dépression ont été respectivement évaluées par la State-Trait Anxiety Inventory (STAI-A \& STAI-B; [21]) et la Beck Depression Inventory (BDI; [22]). Nous n'avons inclus que les patients atteints d'un trouble modérément sévère ( $Y$-BOCS entre 16 et 25 ) avec des symptômes prédominants de vérification. Le type de traitement pharmacologique ne constituait pas un critère d'exclusion mais il devait être stable durant au moins les deux mois précédant leur inclusion. Les patients ne répondant pas aux critères d'inclusion étaient réorientés vers un suivi adapté. 
Les trente cinq patients à qui nous avons proposé de participer à la recherche ont accepté. Quatre n'ont pas terminé leur thérapie et n'ont pas été inclus dans l'analyse : promotion professionnelle $(N=1)$, grossesse $(N=2)$, refus du $3^{\text {ème }}$ examen IRM $(N=1)$. Des absences répétées aux séances de TCC (deux absences consécutives ou trois et plus au cours de la thérapie) constituaient un critère d'exclusion. L'échantillon final est donc de $N=31$ patients. Tous ont donné leur consentement éclairé par écrit pour participer à cette étude sous promotion INSERM, approuvée par un comité de protection des personnes ("Modification of Cerebral Activity of Obsessive Compulsive Disorder (OCD) Patients During Cognitive and Behavioral Therapy (TOC TOC)”, URL: http://clinicaltrials.gov/ct2/show/NCT01331876).

\section{Design de l'étude}

Les patients ont été évalués à quatre moments : immédiatement avant une TCC, à mi-thérapie (env. 1,5 mois après l'examen initial), à la fin de la thérapie ( 3 mois après l'examen initial) et 6 mois après la fin de la thérapie. Chaque session consistait en une évaluation psychiatrique réalisée par un psychologue clinicien n'effectuant pas le suivi thérapeutique des patients ainsi qu'un examen neurocomportemental (voir ci-dessous). Cette recherche s'inscrivait dans le cadre d'un essai clinique contrôlé randomisé en double insu visant à comparer l'efficacité d'une TCC «standard» à celle d'une TCC dite « expérimentale ». Cette dernière est définie par l'adjonction d'un outil psychopédagogique informatisé ciblant spécifiquement le symptôme de vérification.

\section{Thérapies cognitives et comportementales}

Quatre psychologues experts du TOC et de la TCC ont suivi les patients durant 3 mois (15 séances) à l'hôpital de la Pitié-Salpêtrière à raison d'une séance individuelle de 45 minutes par semaine pour les deux types de TCC (standard et expérimentale). Les séances de thérapies étaient financées pendant toute la durée du protocole. Nous nous sommes engagés à orienter vers une prise en charge adaptée les patients pour qui un suivi thérapeutique restait nécessaire à l'issue du protocole.

Avant que les thérapies ne débutent, plusieurs réunions ont été programmées afin d'homogénéiser les pratiques des psychologues. Ces rencontres ont été primordiales pour définir un cadre commun et limiter d'éventuels biais liés aux différences inter-thérapeutes dans « l'application » de la thérapie. Nous avons choisi de nous référer au manuel de Martine Bouvard [23] et d'établir un document résumant de manière concrète et chronologique le déroulement de chaque séance de thérapie.

Après avoir établi un consensus sur la manière de mener les TCC "standards ", nous avons organisé plusieurs ateliers afin de spécifier la nature, les objectifs et l'utilisation de l'outil psychopédagogique informatisé qui caractérise la thérapie dite "expérimentale». Il a été décidé que l'outil serait utilisé systématiquement de la troisième à la quatorzième séance. Les deux premières séances permettaient une prise de contact, une évaluation des troubles et le début d'une alliance thérapeutique. La dernière séance permettait de faire un bilan sur l'évolution clinique du patient et ses projections dans l'avenir. Lors d'un dernier atelier, nous avons testé ensemble l'utilisation pratique de l'outil.

\section{Description de l'outil psychopédagogique informatisé}

Notre outil psychopédagogique est un exercice informatisé de mémoire visuelle dérivé d'un protocole expérimental visant à reproduire et étudier le symptôme de vérification dans un environnement contrôlé [24]. Le patient voit apparaître à l'écran un premier dessin, puis après un délai, un second dessin (figure $1 \mathrm{~A}$ ). Le patient doit indiquer en pressant des boutons réponses si les deux dessins sont identiques (bouton $\mathrm{V}$ ) ou différents (bouton $\mathrm{F}$ ). Immédiatement après avoir donné sa réponse, le patient doit choisir entre deux options : revoir les deux dessins autant de fois qu'il le souhaite pour éventuellement changer sa réponse (vérification) ou confirmer son choix. La confirmation du choix conduit à l'apparition du feedback lui indiquant si sa réponse est juste ou fausse. 
Figure 1 : Paradigmes comportementaux. Panneau A : Outil psychopédagogique. Panneau B : Tâche de provocation de symptômes effectuée en IRMf.

A)

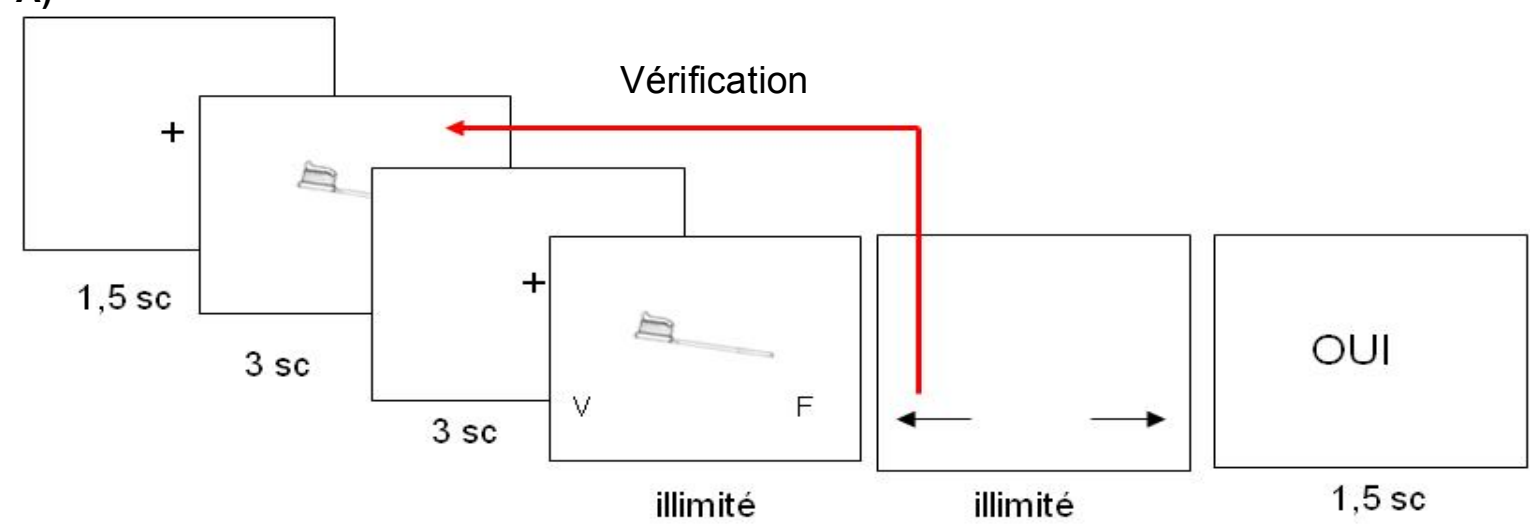

B)

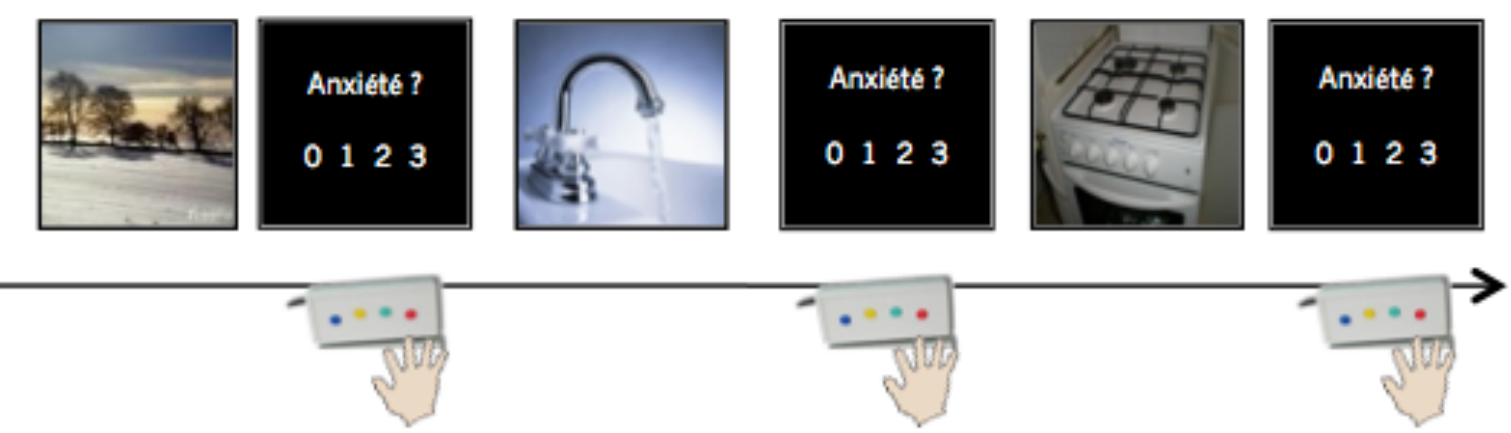

Cet outil a une dimension psychopédagogique car il reproduit les différentes étapes de la vérification (choix - doute - comportement de vérification). En montrant que le nombre de bonnes réponses ne dépend pas du nombre de vérification [24] le thérapeute aide le patient à comprendre que la vérification ne protège pas de l'erreur.

Cet outil est utilisé comme exercice de confrontation virtuelle au doute et de prévention de la réponse dans un contexte non perturbé par l'anxiété ressentie en situation réelle, particulièrement pour les patients les plus réfractaires à l'exposition. Au fil des séances, le thérapeute demande au patient d'essayer de réduire le nombre de vérifications et de tolérer le doute.

L'outil était utilisé durant dix minutes à la fin des séances de thérapie (présentation de huit paires de dessins).

Analyse des données cliniques quantitatives et qualitatives

Nous quantifions l'amélioration clinique comme la différence relative (en \%) des scores entre deux évaluations $(\triangle \mathrm{Y}$-BOCS). Nous évaluons les changements entre deux moments de la thérapie par des $t$-test appariés et, si nécessaire, le $d$ de Cohen et l'intervalle de confiance à $95 \%$ entre crochets. La dynamique temporelle est soumise à des analyses de variance (ANOVA) à mesures répétées avec comme facteur la session d'évaluation (4 niveaux : avant, pendant, après, 6 mois après).

À l'issue du protocole, nous avons interrogé les thérapeutes en suivant un questionnaire semi-structuré portant sur leur vécu du dispositif, l'acceptabilité et l'utilisabilité de l'outil psychopédagogique informatisé afin de compléter les données quantitatives par une approche qualitative. Nous en avons extraits les verbatim les plus représentatifs. 
Paradigme neurocomportemental : Passation d'une tâche de provocation de symptômes personnalisée

Pour rechercher les marqueurs de réponse à la thérapie, nous avons élaboré une tâche comportementale de provocation de symptômes qui consiste à présenter aux patients des stimuli induisant des obsessions et à mesurer leur niveau d'anxiété.

En collaboration avec les chercheurs cliniciens et expérimentalistes, les patients et leurs proches regroupés au sein de l'AFTOC ont été impliqués comme acteurs dans l'élaboration de cette tâche au travers de groupes de paroles. Les patients étaient invités à donner leur avis en tant qu'experts. Ces discussions ont conclu à l'intérêt d'utiliser des photographies individualisées (plutôt que des scénarios génériques lus à haute voix, par exemple). Il s'agissait, pour chaque patient, de photographies prises par lui-même de scènes de son environnement quotidien déclenchant des symptômes.

Outre ces images anxiogènes personnalisées, deux autres catégories de photographies étaient présentées : 1) des scènes neutres (p. ex. paysages, mobilier) ; 2) des scènes dites " génériques ", c'està-dire non-personnalisées et communes à tous les patients, choisies pour induire des obsessions de vérification (p. ex. une gazinière, une porte), figure 1B. Toutes les photographies neutres et génériques ont été sélectionnées par un psychologue clinicien $(M M)$, validées par un groupe de patients indépendants de l'étude et normalisées pour la luminance et le contraste. La tâche était composée de 45 photographies présentées dans un ordre aléatoire : 3 neutres, 3 génériques, 3 personnalisées répétées 5 fois. À chaque image, les patients devaient indiquer leur niveau d'anxiété sur une échelle de 0 (pas d'angoisse) à 3 (anxiété maximum).

Les acquisitions IRM s'effectuaient à l'Institut du Cerveau et de la Moelle épinière. À chacune des quatre sessions, les patients subissaient un examen divisé en plusieurs séquences dont une IRMf visant à enregistrer leur activité cérébrale pendant la tâche de provocation de symptômes [18].

\section{Résultats}

\section{1) Résultats cliniques}

\section{1) Résultats quantitatifs}

Les données démographiques, les scores cliniques à l'inclusion et les données de traitement pharmacologiques sont fournis pour chaque patient dans le Tableau 1. D'un point de vue clinique, les deux groupes de TCC (standard vs expérimentale) ne différaient pas en terme de sévérité initiale (score Y-BOCS : 24,6 ET 4,4 vs 26,2 ET 5,0; $P=0,34$ ). Nous n'avons observé aucune différence dans leur amélioration clinique ( $\triangle \mathrm{Y}$-BOCS : $48 \%$ vs $45 \% ; P=0,36 ; d=0,12)$. Par conséquent, les patients des deux groupes ont été regroupés pour la suite des analyses. 
Tableau 1 : Données démographiques, traitements pharmacologiques et scores cliniques individuels

\begin{tabular}{|c|c|c|c|c|c|c|}
\hline Patient & $\begin{array}{l}\text { Âge } \\
\text { à l'inclusion }\end{array}$ & Sexe & $\begin{array}{l}\text { Âge du } \\
\text { début des } \\
\text { troubles }\end{array}$ & $\begin{array}{l}\text { Durée } \\
\text { du } \\
\text { trouble }\end{array}$ & Traitement pharmacologique & $\begin{array}{l}\text { Score } \\
\text { Y-BOCS à } \\
\text { l'inclusion }\end{array}$ \\
\hline P01 & 45 & $\mathrm{~F}$ & 6 & 39 & - & 20 \\
\hline P03 & 33 & $\mathrm{~F}$ & 16 & 17 & IRSS & 26 \\
\hline P04 & 50 & $\mathrm{~F}$ & 7 & 43 & IRS & 26 \\
\hline P05 & 39 & $\mathrm{~F}$ & 24 & 15 & IRS & 24 \\
\hline P06 & 44 & $\mathrm{~F}$ & 14 & 30 & IRS & 20 \\
\hline P07 & 53 & M & 6 & 47 & $\begin{array}{l}\text { amitryptiline, benzodiazépine } \\
\text { **amitryptiline, benzodiazépine, } \\
\text { clomipramine }\end{array}$ & 25 \\
\hline P08 & 29 & M & 23 & 3 & IRS & 22 \\
\hline P09 & 34 & $\mathrm{~F}$ & 28 & 6 & IRSNA & 21 \\
\hline P10 & 40 & $\mathrm{~F}$ & 12 & 28 & - & 21 \\
\hline P11 & 52 & $M$ & 40 & 12 & - & 21 \\
\hline P12 & 23 & $\mathrm{~F}$ & 13 & 10 & - & 25 \\
\hline P13 & 37 & $\mathrm{~F}$ & 6 & 31 & - & 24 \\
\hline P14 & 35 & $\mathrm{~F}$ & 6 & 29 & - & 21 \\
\hline P15 & 24 & M & 10 & 14 & IRSS & 21 \\
\hline P16 & 30 & M & 24 & 6 & $\begin{array}{l}\text { IRSS, neuroleptique } \\
* * \text { IRSS, benzodiazépine }\end{array}$ & 33 \\
\hline P17 & 33 & M & 28 & 5 & - & 28 \\
\hline P18 & 22 & M & 10 & 12 & $\begin{array}{l}\text { clomipramine, benzodiazépine } \\
{ }^{* *} \text { IRSS, neuroleptique atypique, } \\
\text { valproate }\end{array}$ & 20 \\
\hline P20 & 35 & $\mathrm{~F}$ & 23 & 16 & IRSS, benzodiazépine & 30 \\
\hline P21 & 38 & M & 23 & 15 & - & 28 \\
\hline P23 & 27 & M & 11 & 16 & $\begin{array}{l}\text { IRSS, benzodiazépine } \\
\text { *IRSS, lithium, neuroleptique, } \\
\text { clomipramine }\end{array}$ & 30 \\
\hline P24 & 55 & $\mathrm{~F}$ & 23 & 32 & $\begin{array}{l}\text { IRSS, benzodiazépine, } \\
\text { neuroleptique } \\
* * \text { IRSS, benzodiazépine, } \\
\text { neuroleptique, clomipramine }\end{array}$ & 28 \\
\hline P26 & 38 & M & 20 & 18 & - & 21 \\
\hline P27 & 22 & $\mathrm{~F}$ & 16 & 6 & $\begin{array}{l}\text { neuroleptique atypique, } \\
\text { clomipramine } \\
{ }^{* *} \text { neuroleptique atypique, IRSS }\end{array}$ & 33 \\
\hline P28 & 31 & $\mathrm{~F}$ & 7 & 24 & - & 33 \\
\hline P29 & 24 & $\mathrm{~F}$ & 11 & 13 & - & 27 \\
\hline P30 & 56 & M & 34 & 22 & $\begin{array}{l}\text { neuroleptique, clomipramine, } \\
\text { benzodiazépine } \\
{ }^{* *} \text { clomipramine, } \\
\text { benzodiazépine, antidépresseur } \\
\text { tétracyclique }\end{array}$ & 21 \\
\hline P31 & 26 & $M$ & 16 & 10 & - & 22 \\
\hline P32 & 33 & $\mathrm{~F}$ & 23 & 10 & SNRI, valproate & 28 \\
\hline P33 & 39 & $\mathrm{~F}$ & 29 & 10 & - & 20 \\
\hline P34 & 53 & M & 18 & 35 & $\begin{array}{l}\text { IRS, benzodiazépine } \\
{ }^{* *} \text { neuroleptique atypique }\end{array}$ & 36 \\
\hline P35 & 35 & $\mathrm{~F}$ & 8 & 27 & - & 31 \\
\hline
\end{tabular}

Abréviations : IRS : Inhibiteur de Recapture de la Sérotonine ; IRSS : Inhibiteur de Recapture Spécifique de la Sérotonine ; IRSNA : Inhibiteur de Recapture de la Sérotonine et de la Noradrénaline.

- pas de traitement

* changement de traitement au cours de la TCC

** changement de traitement après la TCC

La TCC a amélioré les symptômes du TOC de 46,3\%, $d=1,76[1,172,34]$ à l'issue de la thérapie. Dès la mi-thérapie, cette amélioration atteignait déjà $29,5 \%$. En revanche, l'amélioration s'est interrompue en 
post-thérapie : 8,2\% (Effet session: $F(3,90)=65,7, P<0,001$ ). La dépression et l'anxiété se sont également améliorées pour la BDI : $-31 \%$ à la fin de la thérapie et $-35 \%$ six mois après $(P<0,005)$; pour la STAI-A : $-19 \%$ à la fin de la thérapie et $-9 \%$ six mois après $(P<0,005)$; pour la STAI-B : $-12 \%$ à la fin de la thérapie et $-11 \%$ six mois après $(P<0,005)$, voir Tableau 2 .

Tableau 2: Données cliniques et démographiques des patients

\begin{tabular}{|c|c|c|c|c|c|c|c|}
\hline \multirow[b]{2}{*}{ Echelles } & \multirow{2}{*}{$\begin{array}{c}\text { Avant } \\
\text { thérapie } \\
\text { Score } \\
\text { moyen } \\
\text { (écart- } \\
\text { type) }\end{array}$} & \multicolumn{2}{|c|}{ À mi-thérapie } & \multicolumn{2}{|c|}{ Après thérapie } & \multicolumn{2}{|c|}{ Six mois après thérapie } \\
\hline & & $\begin{array}{l}\text { Score } \\
\text { moyen } \\
\text { (écart- } \\
\text { type) }\end{array}$ & $\begin{array}{l}\text { Evolution } \\
\text { (base : } \\
\text { «avant») }\end{array}$ & $\begin{array}{l}\text { Score } \\
\text { moyen } \\
\text { (écart- } \\
\text { type) }\end{array}$ & $\begin{array}{c}\text { Evolution } \\
\text { (base : } \\
\text { «avant })\end{array}$ & $\begin{array}{c}\text { Score } \\
\text { moyen } \\
\text { (écart- } \\
\text { type) }\end{array}$ & $\begin{array}{l}\text { Evolution } \\
\text { (base : } \\
\text { « après ») }\end{array}$ \\
\hline Y-BOCS & $\begin{array}{l}25,4 \\
(4,7)\end{array}$ & $18,5(6,5)$ & $P<0,005$ & $\begin{array}{l}14,1 \\
(7,7)\end{array}$ & $P<0,005$ & $\begin{array}{c}14,2 \\
(10,0)\end{array}$ & n.s. \\
\hline BDI & $\begin{array}{l}10,8 \\
(6,4)\end{array}$ & \multicolumn{2}{|c|}{ Non mesuré } & $\begin{array}{c}7,4 \\
(4,9)\end{array}$ & $P<0,005$ & $\begin{array}{c}7,0 \\
(4,9)\end{array}$ & n.s. \\
\hline STAI A & $\begin{array}{c}46,8(13, \\
6)\end{array}$ & \multicolumn{2}{|c|}{ Non mesuré } & $\begin{array}{c}37,7 \\
(10,3)\end{array}$ & $P<0,005$ & $\begin{array}{c}42,4 \\
(15,5)\end{array}$ & n.s. \\
\hline STAI B & $\begin{array}{c}57,7 \\
(10,6)\end{array}$ & \multicolumn{2}{|c|}{ Non mesuré } & $\begin{array}{l}50,5 \\
(9,5)\end{array}$ & $P<0,005$ & $\begin{array}{c}51,2 \\
(13,2)\end{array}$ & n.s. \\
\hline
\end{tabular}

Patients : $\mathrm{N}=31$; âge moyen $=36,6$ ans.

Note : Deux patients n'ont pas effectué leur examen 6 mois après thérapie.

n.s. non significatif

\section{1) Résultats qualitatifs}

Concernant l'outil psychopédagogique informatisé, tous les thérapeutes le trouvent facile d'utilisation. Un seul est plus nuancé : "J'ai trouvé le logiciel simple à utiliser mais allumer le pc est très contraignant et je n'ai pas trouvé que cela remplaçait fondamentalement une exposition ou une bonne vieille explication sur le cerveau. ". Les thérapeutes se sont bien approprié la démarche et le discours associés à l'outil: "Je me suis sentie plus à l'aise avec la thérapie expérimentale avec la psychopédagogie qu'avec la thérapie classique ». Ils précisent comment l'outil est venu soutenir leur discours : "L'outil informatique peut être un bon exemple illustratif du mécanisme inadapté de la vérification. La personne peut se rendre compte par sa propre expérience que la vérification importante n'est pas associée à un évitement de l'erreur. Elle peut donc vivre dans une situation qui l'implique moins émotionnellement [...]. Cet exercice vient étayer toutes les explications de la boucle neurobiologique expliquée lors de la deuxième séance. ".

La principale limite de l'outil est l'adhésion non systématique des patients. Un thérapeute rapporte un potentiel effet de renforcement pour un patient qui, après plusieurs séances de thérapie arrive à moins vérifier mais fait plus d'erreurs : les vérifications pouvant alors être perçues comme bénéfiques.

Enfin, les patients ont le plus souvent apprécié l'outil "ludique mais sérieux", comme une clôture distrayante des séances. Comme escompté, l'outil semble permettre une meilleure compréhension du trouble et d'acquérir la capacité de s'exposer à des situations anxiogènes. L'un des patients rapporte ainsi à son thérapeute : "Devant ma porte, j'ai pensé à l'ordinateur. ".

Les thérapeutes ont souligné le contexte particulier de l'utilisation de cet outil dans le cadre d'une recherche standardisée : "J'ai vécu de la frustration de ne pas utiliser tout mon panel d'outils avec les patients, devant me limiter à certaines stratégies et discours selon les groupes. ».

Les thérapeutes notent que cette thérapie dont la fin est programmée permet aux patients de se mobiliser davantage que dans un processus thérapeutique "sans fin». Ils expliquent que cette 
expérience a enrichi leur pratique habituelle, qu'ils axent désormais davantage les séances de thérapies sur la psychoéducation.

\section{2) Résultats comportementaux à la tâche de provocation de symptômes}

Au cours de la tâche de provocation de symptômes, l'anxiété rapportée par les patients différait de façon significative en fonction du type de photographies $(F(2,52)=57,8, P<0,001)$. Sur toute la durée du protocole, les images personnalisées ont été jugées plus anxiogènes que les génériques qui étaient elles-mêmes plus anxiogènes que les neutres (cotations moyennes respectives : 1,$43 ; 0,85 ; 0,21$ ). À l'issue de la thérapie, l'anxiété rapportée par les patients a diminué pour tous les types de photographies. Cependant, cette diminution était significativement plus importante pour les photographies personnalisées, que pour les génériques et les neutres (respectivement - $28,2 \%$; -20,41\% ; $6,24 \% ; F(6,156)=13,24, P<0,001)$. Néanmoins, nous n'avons retrouvé aucun effet de la thérapie sur l'anxiété rapportée dans la tâche, ni comme effet principal $(F(1,26)=0.02, P=0,9)$, ni dans l'interaction avec les autres facteurs (tous les $F<1$; tous les $P>0,5$ ). Voir figure $2 A \& 2 B$.

Figure 2 : Résultats cliniques (panneau A) et neurocomportementaux (panneau B).
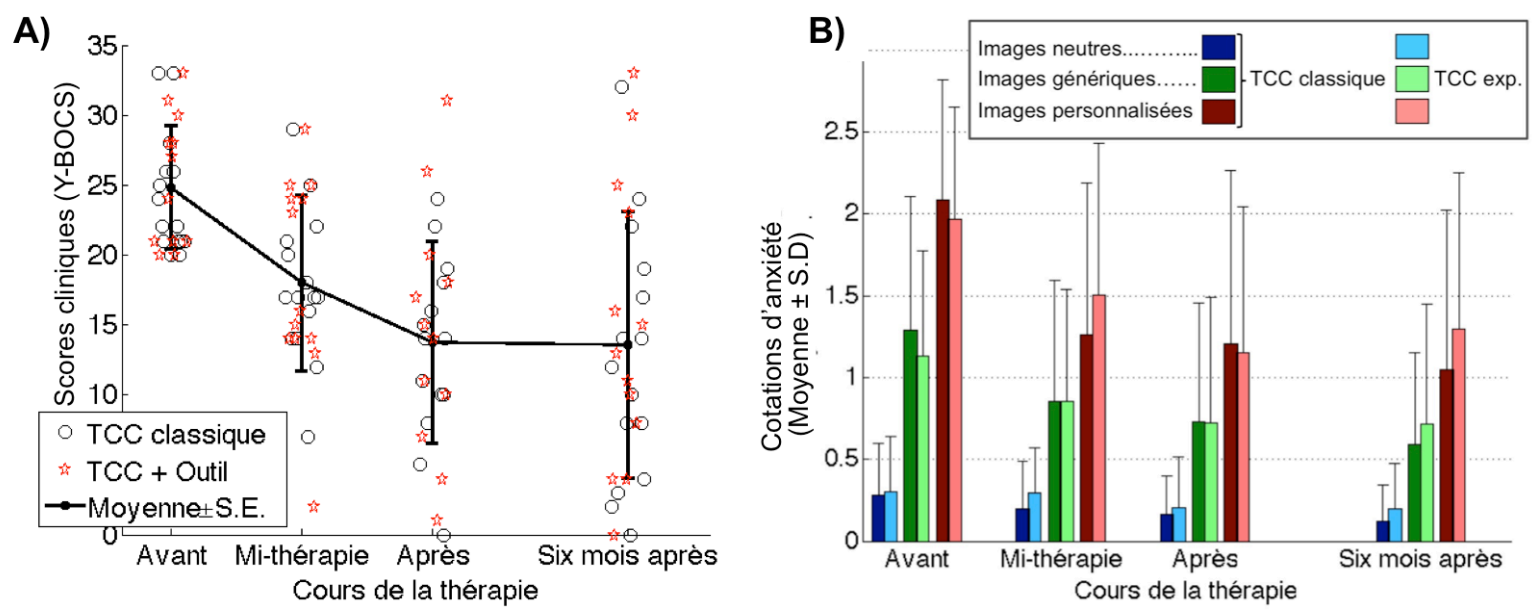

\section{3) Prédicteurs de réponse}

Compte tenu de l'amélioration clinique à mi-thérapie, nous avons étudié la corrélation entre l'amélioration après thérapie et les indicateurs suivants à mi-thérapie (i) l'amélioration clinique $(\Delta Y$ BOCS) et (ii) la diminution des cotations d'anxiété lors de la présentation des photographies.

Ces analyses révèlent (i) une corrélation forte entre l'amélioration clinique intermédiaire et l'amélioration après thérapie : $r=0,82, P<0,001$; (ii) une corrélation moyenne significative entre l'amélioration clinique et la diminution de l'anxiété liée aux photographies personnalisées $r=0,42, p<$ 0,05 .

\section{Discussion}

Nous avons conçu un outil psychopédagogique informatisé en nous appuyant sur des travaux expérimentaux en recherche clinique et en concertation avec les thérapeutes et les patients dans une démarche participative. Cet outil conçu comme un adjuvant à la TCC standard confrontait le patient à un exercice durant lequel il pouvait effectuer des vérifications tout en recevant un feedback sur sa performance. Les thérapeutes se sont bien appropriés cet outil. La TCC étant déjà efficace selon les critères de la médecine fondée sur les preuves (EBM), le gain thérapeutique de cette adjonction s'est toutefois relevé non-significatif. 
L'absence de différence d'efficacité entre les TCC «standard » et « expérimentale » peut se comprendre à la lumière des théories de Wampold [25], pour qui la variance des résultats dû à la psychothérapie peut se répartir ainsi : 13 \% dus à la psychothérapie elle-même (lesquels se partagent en $70 \%$ liés aux «facteurs communs » à savoir l'alliance thérapeutique, l'allégeance, l'adhérence et le thérapeute, 22\% aux facteurs non-contrôlés du patient et $8 \%$ aux «facteurs spécifiques ») et $87 \%$ à d'autres facteurs extérieurs à la psychothérapie. L'adjonction d'un outil psychopédagogique à une TCC s'inscrit dans ces facteurs spécifiques qui représentent seulement $1 \%$ de la variance totale de l'efficacité. Ainsi, d'après la taille d'effet observée a posteriori $(d=0,12)$, un effectif de $N=860$ patients serait nécessaire pour atteindre la puissance suffisante à la démonstration statistique d'une augmentation de l'efficacité thérapeutique liée à notre outil. Fort de ces constatations, plusieurs voies de recherche restent ouvertes pour améliorer la prise en charge des patients souffrant de TOC. Il s'agirait notamment d'identifier des sous-groupes de patients susceptibles de bénéficier optimalement d'innovations telles que notre outil informatisé [26] [27]. Une alternative radicale consisterait à changer de paradigme [28], à s'intéresser plutôt aux «facteurs non-spécifiques» de façon à mieux les spécifier pour potentialiser leur intérêt thérapeutique.

Nos résultats tendent à confirmer l'efficacité thérapeutique de la TCC dans le TOC [2] [29] sans prétendre toutefois à la démontrer selon les normes EBM. En l'absence de groupe contrôle, e.g. waiting list, il n'est pas possible d'exclure un possible effet de retour à la moyenne. Pour autant, ces résultats ne peuvent pas être directement transposés à la pratique thérapeutique quotidienne en raison des contraintes inhérentes aux études contrôlées randomisées [30] [31] : les patients de cette étude étaient cliniquement homogènes et sans comorbidité (cf. Méthode). Cela diffère de la population typique des patients atteints de TOC, 50\% d'entre eux ayant au moins une comorbidité notamment dépressive ou anxieuse [32] [29]. Malgré le niveau faible de ces deux dimensions à l'inclusion, la TCC axée sur le TOC permet une réduction de la symptomatologie anxieuse et dépressive [33]. À un autre niveau, la standardisation des protocoles thérapeutiques pour les besoins de la recherche impose un cadre strict qui ne correspond pas toujours à la clinique. Les thérapeutes ont exprimé leur difficulté à maintenir le cadre des traitements, uniquement axés sur la prise en charge du TOC, alors que certains patients vivaient pendant cette étude des événements difficiles. Le fait de se référer à un même manuel et de suivre scrupuleusement ses prescriptions (adhérence) permet de standardiser les thérapies et de diminuer les biais liés à la variabilité inter-thérapeutes dans l'application du traitement. Cependant, l'adhérence peut avoir un effet négatif, en limitant l'exercice de la compétence du thérapeute, c'est-à-dire sa capacité à s'adapter à la situation précise du patient [32].

L'introduction de l'outil psychopédagogique a nécessité que les thérapeutes s'attachent à une technique prédéfinie, ce qui peut nuire à la qualité de l'alliance thérapeutique [34] [5]. Néanmoins, dans le traitement de la dépression, il a été montré que la TCC assistée par ordinateur est bien acceptée par les patients, cliniquement efficace et pertinente même en pratique courante [36] [37] [38]. L'un des défis à relever pour les études de thérapie cognitive assistée par ordinateur est de vérifier que l'efficacité des essais contrôlés peut être efficiente, c'est-à-dire se transposer aux soins courants.

Sur la base de la littérature, nous avons conçu une tâche de provocation de symptômes [16] [39] au sein de laquelle nous avons employé des photographies personnalisées dans le but d'induire des symptômes plus forts qu'avec les images génériques. Nos résultats comportementaux démontrent que les photographies personnalisées sont plus sensibles que les génériques, validant leur utilisation dans un cadre expérimental. Des photographies génériques peuvent être insuffisantes pour induire une obsession car les obsessions et les compulsions dépendent souvent de l'environnement immédiat des patients. Dans cette perspective, les techniques de réalité virtuelle [40] ou augmentée [41] offrent une alternative prometteuse. Dans l'article associé [18] nous avions observé que la tâche de provocation de symptômes activait des régions cérébrales connues pour leur hyperactivité associée au TOC (cortex cingulaire antérieur et orbitofrontal) [13] [15] [24]. L'activation de ces régions diminuait au cours de la thérapie sans toutefois pouvoir être corrélée à l'amélioration individuelle des patients. La corrélation entre la 
diminution des scores d'anxiété dans la tâche comportementale et l'amélioration clinique, bien qu'obtenue dans le contexte particulier d'un protocole de recherche (qui plus est en IRM), garde un intérêt comme instrument d'évaluation clinique. Ces résultats invitent a explorer l'intérêt que peuvent avoir des tâches neurocomportementales utilisant des stimuli personnalisés pour le pronostic de l'efficacité thérapeutique de la TCC dans le TOC.

\section{Conclusion}

Bien que notre outil psychopédagogique informatisé soit bien accepté tant pas les patients que les thérapeutes, il n'apporte pas de gain d'efficacité mesurable par rapport à une TCC standard. Si on est tenté de répondre «Non! » à l'intitulé de cet article, d'autres perspectives peuvent être considérées. Dans une optique de soins personnalisés, il ne s'agirait plus de rechercher une efficacité pour un patient « moyen " mais de caractériser les patients susceptibles de bénéficier au mieux d'innovations thérapeutiques. Dans une optique d'optimisation de la TCC, nos résultats suggèrent qu'il faudrait explorer les premières étapes du processus thérapeutique pour pouvoir ajuster les stratégies thérapeutiques précocement. Dans une optique de médecine mentale fondée sur les preuves (EBM), vouloir augmenter l'efficacité d'une thérapie qui bénéficie du plus haut niveau de preuves est un défi. Dans une vision holiste de la personne, l'objectif ne serait plus de diminuer l'intensité des symptômes mais leur impact sur la qualité de vie. La question serait alors : "Peut-on diminuer le retentissement du trouble obsessionnel compulsif par un adjuvant informatique innovant ?». Et du point de vue de l'économie de la santé, la question pourrait se poser selon un critère d'efficience : "Jusqu'où faut-il chercher à augmenter l'efficacité de la thérapie cognitive et comportementale pour le trouble obsessionnel compulsif par un adjuvant informatique innovant?».

\section{Déclaration d'intérêts}

Les auteurs déclarent ne pas avoir de conflits d'intérêt en relation avec cet article.

\section{Remerciements}

Ce projet a été financé en 2008 par la région lle de France dans le cadre d'un projet PICRI (Partenariat Institution-Citoyens pour la Recherche et l'Innovation).

Nous remercions Christophe Demonfaucon et les membres de l'AFTOC pour leur aide et leur soutien à toutes les étapes de l'étude. Nous remercions également Vincent Trybou, Anna Maestre, Corine Lobjoie, et Romina Bianco pour avoir mené des séances de TCC, ainsi que Aurélie Saulton et Christian Gaudeau pour avoir réalisé les procédures d'IRM. Nous remercions Bruno Falissard pour l'aide à la conception de l'étude, Benjamin Granger pour les analyses statistques et Vincent Morice pour la procédure de randomisation, et Xavier Briffault pour ses dé-façonnages.

\section{Références}

1. Ruscio AM, Stein DJ, Chiu WT, et al. The epidemiology of obsessive-compulsive disorder in the National Comorbidity Survey Replication. Mol Psychiatry 2010;15(1): 53-63.

2. Gava I, Barbui C, Aguglia E, et al. Psychological treatments versus treatment as usual for obsessive compulsive disorder (OCD). Cochrane Database Syst Rev 2007;(2): CD005333.

3. Mallet $L$, Polosan $M$, Jaafari $N$, et al. Subthalamic nucleus stimulation in severe obsessive-compulsive disorder. N Engl J Med 2008;359(20): 2121-34.

4. Marks IM, Cuijpers P, Cavanagh K, et al. Meta-analysis of computer-aided psychotherapy: problems and partial solutions. Cogn Behav Ther 2009;38(2): 83-90. 
5. Lind C, Boschen MJ, Morrissey S. Technological advances in psychotherapy: implications for the assessment and treatment of obsessive compulsive disorder. J Anxiety Disord 2013;27(1): 47-55.

6. Lovell K, Bee P. Optimising treatment resources for OCD: a review of the evidence base for technology-enhanced delivery. J Ment Health 2011;20(6): 525-42.

7. Meyerbroker K, Emmelkamp PM. Virtual reality exposure therapy in anxiety disorders: a systematic review of process-and-outcome studies. Depress Anxiety 2010;27(10): 933-44.

8. Jonsson $\mathrm{H}$, Hougaard $\mathrm{E}$, Bennedsen $\mathrm{BE}$. Randomized comparative study of group versus individual cognitive behavioural therapy for obsessive compulsive disorder. Acta Psychiatr Scand 2011;123(5): 387-397.

9. Baxter LR, Saxena S, Brody AL, et al. Brain Mediation of Obsessive-Compulsive Disorder Symptoms: Evidence From Functional Brain Imaging Studies in the Human and Nonhuman Primate. Semin Clin Neuropsychiatry 1996;1(1): 32-47.

10. Schwartz JM. Neuroanatomical aspects of cognitive-behavioural therapy response in obsessive-compulsive disorder. An evolving perspective on brain and behaviour. $\mathrm{Br} \mathrm{J}$ Psychiatry Suppl 1998;(35): 38-44.

11. Linden M. Minimal emotional dysfunctions (MED) in personality disorders. European Psychiatry 2006;21(5): 325-332.

12. Schiepek G, Tominschek I, Karch S, et al. A controlled single case study with repeated fMRI measurements during te treatment of a patient with obsessive-compulsive disorder: testing the nonlinear dynamics approach to psychotherapy. World J Biol Psychiatry 2009;10(4 Pt 2): 658-68.

13. Aouizerate $B$, Rotge JY, Bioulac $B$, et al. Present contribution of neurosciences to a new clinical reading of obsessive-compulsive disorder. Encephale 2007;33(2): 203-10.

14. Clark DA, Beck AT. Cognitive theory and therapy of anxiety and depression: convergence with neurobiological findings. Trends Cogn Sci 2010;14(9): 418-24.

15. Linden DE. How psychotherapy changes the brain-the contribution of functional neuroimaging. Mol Psychiatry 2006;11(6): 528-38.

16. Rauch SL, Jenike MA, Alpert NM, et al. Regional cerebral blood flow measured during symptom provocation in obsessive-compulsive disorder using oxygen 15-labeled carbon dioxide and positron emission tomography. Arch Gen Psychiatry 1994;51(1): 62-70.

17. Cottraux J. Psychotherapies: what is the impact on the cortical and subcortical levels?. Encephale 2006;32(Pt 2): S48-52.

18. Morgiève M, N'Diaye K, Haynes WIA, et al. Dynamics of psychotherapy-related cerebral haemodynamic changes in obsessive compulsive disorder using a personalized exposure task in functional magnetic resonance imaging. Psychol Med 2014;44(7) 1461-73.

19. Sheehan DV, Lecrubier Y, Sheehan KH, et al. The Mini-International Neuropsychiatric Interview (M.I.N.I.): the development and validation of a structured diagnostic psychiatric interview for DSM-IV and ICD-10. J Clin Psychiatry 1998;59 Suppl 20: 2233; quiz 34-57.

20. Goodman WK, Price LH Rasmussen SA, et al. The Yale-Brown Obsessive Compulsive Scale. I. Development, use, and reliability. Arch Gen Psychiatry 1989;46(11): 1006-11.

21. Spielberger C.. Manual for the State-Trait Anxiety Inventory (form Y). Mind Garden, Redwood City, CA, USA ; 1983.

22. Beck AT, Beamesderfer A. Assessment of depression: the depression inventory. Mod Probl Pharmacopsychiatry 1974;7(0): 151-69.

23. Bouvard M. Les troubles obsessionnels compulsifs : Principes, thérapies, applications. Masson, Paris ; 2006.

24. Rotge JY, Guehl D, Dilharreguy B et al. Provocation of obsessive-compulsive symptoms: a quantitative voxel-based meta-analysis of functional neuroimaging studies. J Psychiatry Neurosci 2008b;33(5): 405-12.

25. Wampold BE, Mondin GW, Moody M, et al. A meta-analysis of outcome studies comparing bona fide psychotherapies: Empiricially, "all must have prizes.". Psychol Bull 1997;122(3): 203-215. 
26. Beevers CG, McGeary JE. Therapygenetics: moving towards personalized psychotherapy treatment. Trends Cogn Sci 2012;16(1): 11-2.

27. Simon GE, Perlis RH. Personalized medicine for depression: can we match patients with treatments? Am J Psychiatry 2010;167(12): 1445-55.

28. Castelnuovo G. Empirically supported treatments in psychotherapy: towards an evidence-based or evidence-biased psychology in clinical settings? Front Psychol 2010;1: 27.

29. Abramowitz JS, Taylor S, McKay D. Obsessive-compulsive disorder. Lancet 2009;374(9688): 491-9.

30. Laurenceau JP, Hayes AM, Feldman GC. Some methodological and statistical issues in the study of change processes in psychotherapy. Clin Psychol Rev 2007;27(6): 682-95.

31. Morgiève M, Briffault X. La fabrique du TOC moderne- Une analyse ethnographique de la cérébralisation de la névrose obsessionnelle dans un laboratoire de neurosciences cliniques. Socio-logos 2014;9: 1-15.

32. Steketee G, Siev J, Fama J, et al. Predictors of course in obsessive-compulsive disorder. Psychiatry Res 1999;89(3): 229-38.

33. Servant $D$, Germe A, Autuori $M$, et al. An integrative and transdiagnostic relaxation protocol for anxious patients. Results of a pilot study. Encephale 2014.

34. Castonguay LG, Goldfried MR, Wiser S, et al. Predicting the effect of cognitive therapy for depression: a study of unique and common factors. J Consult Clin Psychol 1996;64(3): 497-504.

35. Roth A, Fonagy P. What Works For Whom?: A Critical Review Of Psychotherapy Research Guilford Press, New-York, USA ; 2005.

36. Proudfoot J, Everitt B, Shapiro DA, et al. Clinical efficacy of computerised cognitivebehavioural therapy for anxiety and depression in primary care: randomised controlled trial. Br J Psychiatry 2004;185(1): 46-54.

37. Foroushani PS, Schneider J, Assareh N. Meta-review of the effectiveness of computerised CBT in treating depression. BMC Psychiatry 2011;11: 131.

38. Cuijpers $P$, dekker J, Hollon DSD, et al. Adding psychotherapy to pharmacotherapy in the treatment of depressive disorders in adults: a meta-analysis. J Clin Psychiatry 2009;70(9): 1219-29.

39. Mataix-Cols D, Wooderson S, Lawrenc N, et al. Distinct neural correlates of washing, checking, and hoarding symptom dimensions in obsessive-compulsive disorder. Arch Gen Psychiatry 2004;61(6): 564-76.

40. Kim K, Kim SI, Cha K, et al. Development of a computer-based behavioral assessment of checking behavior in obsessive-compulsive disorder. Compr Psychiatry 2010;51(1): 8693.

41. Breton-Lopez J, Quero S, Botella C, et al. An augmented reality system validation for the treatment of cockroach phobia. Cyberpsychol Behav Soc Netw 2010;13(6): 705-10. 